\title{
Polygon-circle and word-representable graphs
}

\author{
Jessica Enright ${ }^{1}$ \\ Global Academy of Agriculture and Food Security \\ University of Edinburgh, Scotland \\ Sergey Kitaev ${ }^{2}$ \\ Computing and Information Science \\ University of Strathclyde, Scotland
}

\begin{abstract}
We describe work on the relationship between the independently-studied polygoncircle graphs and word-representable graphs.

A graph $G=(V, E)$ is word-representable if there exists a word $w$ over the alphabet $V$ such that letters $x$ and $y$ form a subword of the form $x y x y \cdots$ or yxyx $\cdots$ iff $x y$ is an edge in $E$. Word-representable graphs generalise several well-known and well-studied classes of graphs $[2,3]$. It is known that any word-representable graph is $k$-word-representable, that is, can be represented by a word having exactly $k$ copies of each letter for some $k$ dependent on the graph. Recognising whether a graph is word-representable is NP-complete ([3, Theorem 4.2.15]). A polygoncircle graph (also known as a spider graph) is the intersection graph of a set of polygons inscribed in a circle [4]. That is, two vertices of a graph are adjacent if their respective polygons have a non-empty intersection, and the set of polygons that correspond to vertices in this way are said to represent the graph. Recognising whether an input graph is a polygon-circle graph is NP-complete [5]. We show that neither of these two classes is included in the other one by showing that the wordrepresentable Petersen graph and crown graphs are not polygon-circle, while the non-word-representable wheel graph $W_{5}$ is polygon-circle. We also provide a more
\end{abstract}


refined result showing that for any $k \geq 3$, there are $k$-word-representable graphs which are neither $(k-1)$-word-representable nor polygon-circle.

Keywords: polygon-circle graph, word-representable graph, Petersen graph

\section{Introduction}

Polygon-circle graphs are the intersection graphs of polygons inscribed in a circle, and are one of many well-studied classes of intersection graphs. While recognising whether a graph is a polygon-circle graph is NP-complete, many problems that are NP-Complete in general can be resolved in polynomial time given a polygon-circle representation of a graph $[5,6]$. This algorithmic advantage, as well as the position of the polygon-circle graphs in the broad hierarchy of intersection classes, has been the focus of much of the study on this class. Polygon-circle graphs can also be defined in terms of a string that has a direct correspondence to a polygon-circle representation.

Word-representable graphs are defined primarily in terms of a string representation: we say that two letters $x, y$ alternate in a string $S$ if, when we delete all letters other than $x$ and $y$, the string is either of the form $x y x y \ldots$ or $y x y x \ldots$, of either even or odd length. Word-representable graphs can be characterised by a semi-transitive orientation of their edges (as described in [2], not to be defined here), and generalise the comparability graphs (which are graphs admitting transitive orientations), and the 3-colourable graphs.

It is often of interest to consider polygon-circle graphs of limited polygons, or word-representable graphs of limited numbers of copies of each letter: if a graph is representable by polygons of at most $k$ corners inscribed in a circle, then it is a $k$-polygon-circle graph, and if a graph is word-representable by a string in which each letter appears at most $k$ times, then it is $k$-wordrepresentable. We note that originally $k$-word-representable graphs are defined as graphs admitting representation by a string in which each letter appears exactly $k$ times, but "exactly" here is equivalent to "at most", and in fact for any word-representable graph $G$ there exists $k$ such that $G$ is $k$-wordrepresentable $[2,3]$.

2-polygon-circle graphs are the intersection graphs of chords in a circle, and are also known as circle graphs. These coincide exactly with the

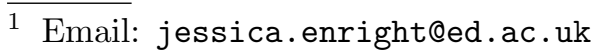

2 Email: sergey.kitaev@cis.strath.ac.uk 
2-word-representable graphs, and so 2-polygon-circle graphs are exactly 2word-representable graphs [2]. Beyond this result, little further was known on interrelations between these two classes of graphs. In this note, we outline progress in understanding the relationship between polygon-circle and wordrepresentable graphs, showing that neither is a subset of the other.

\section{Definitions and preliminaries}

A polygon-circle representation of a graph $G=(V, E)$ is a mapping $\mathcal{P}$ of vertices in $V$ to polygons in a circle such that $(u, v) \in E$ if and only if $\mathcal{P}(u)$ intersects $\mathcal{P}(v)$. Sometimes for convenience we will use subscripting, as in $P_{i}$, to denote the polygon $\mathcal{P}\left(v_{i}\right)$. A corner-string of a polygon-circle representation is a string produced by starting at an arbitrary point on the circle that hosts the representation, and proceeding around the circle, adding a letter denoting the vertex represented by a polygon each time a corner of a polygon encountered (example in Figure 1). Note that a single polygon-circle representation has many possible corner-strings, depending on the starting point. It is known [6] that two polygons representing vertices $u$ and $v$ intersect if any only if, when we delete all corners other than $u$ and $v$ from the cornerstring, the result has as a substring either uvuv or vuvu, in which case we say that $u$ and $v$ loosely alternate, and that the segments overlap. Note that this loose alternation is less strict than the notion of alternation used to define word-representable graphs: in the string $x y x x y x$ the letters $x$ and $y$ loosely alternate, but they do not alternate in the word-representation sense because there are two copies of $x$ between two consecutive copies of $y$.

If a vertex $v_{i}$ is represented by a polygon $P_{i}$ in a polygon-circle representation that gives a corner-string $\mathcal{S}$, then the segment of $u($ denoted $s(u))$ in $\mathcal{S}$ is the minimal contiguous substring of $\mathcal{S}$ that contains all occurrences of $u$.

We say that a segment $s(u)$ is contained in $s(v)$ (denoted $s(u) \subset s(v)$ ) if $s(u)$ is entirely between two consecutive occurrences of $v$. Contrast this to the idea of a subsegment: we say $s(u)$ is a subsegment of $s(v)$ if $s(u)$ is a substring of $s(v)$. Segment containment is transitive and antisymmetric. Given a corner-string $\mathcal{S}$, we say that a segment $s$ is outermost if it is not contained in any other segment. A path from vertex $a$ to $b$ is a sequence of vertices starting with $a$ and ending with $b$ such that each vertex is adjacent to the one before it in the sequence.

We use several relatively straightforward lemmas (proofs omitted for space):

Lemma 2.1 If two segments intersect but their corresponding polygons do 
not, then one is contained in the other.

Lemma 2.2 If $s(u) \subset s(v)$ and $s(v) \subset s(w)$, then all paths from $u$ to $w$ must pass through the neighbourhood of $v$.

Lemma 2.3 In a corner-string from a polygon-circle representation of a chordless cycle on $\geq 4$ vertices at least one vertex's segment contains another.

\subsection{Word-representable graphs}

The wheel graph $W_{n}$ is the graph on $n+1$ vertices obtained from the cycle graph $C_{n}$ by adding an all-adjacent vertex.

Lemma 2.4 ([3]) Wheel graphs $W_{2 n+1}$ are non-word-representable for $n \geq 2$.

Lemma $2.5([2,3])$ The Petersen graph (shown in Figure 2) is 3-word-representable but not 2-word-representable.

A crown graph $H_{n, n}$ is a graph obtained from the complete bipartite graph $K_{n, n}$ by removing a perfect matching. See Figure 2 for $H_{4,4}$.

Lemma 2.6 ([1]) For $n \geq 5$, the crown graph $H_{n, n}$ is $\lceil n / 2\rceil$-word-representable but not $(\lceil n / 2\rceil-1)$-word-representable.

\section{Polygon-circle graphs vs word-representable graphs}

In this section we will show that the word-representable Petersen graph and crown graphs are not polygon-circle, while the non-word-representable wheel graph $W_{5}$ is polygon-circle. We also provide a more refined result showing that for any $k \geq 3$, there are $k$-word-representable graphs which are neither $(k-1)$-word-representable nor polygon-circle.

Lemma 3.1 The wheel graph $W_{n}$ is a polygon-circle graph for $n \geq 3$.

Proof. Essentially, for a wheel $W_{n}$ we represent the vertices on the large outer cycle $v_{1} \ldots v_{n}$ with chords on the circle. We can place their corners starting at an arbitrary point on the circle in the sequence $v_{0}, v_{n}, v_{1}, v_{0}, v_{2}, v_{1}, v_{3}, \ldots v_{n-1}, v_{n}$. Then the central high-degree vertex is represented by a polygon with corners between the first corner of $v_{2}$ and the second corner of $v_{1}$, the first corner of $v_{4}$ and the second corner of $v_{3}$, etc - essentially between every second pair of chord corners, giving this polygon representing the central vertex of the wheel on $n$ vertices $\lceil n / 2\rceil$ corners, producing a $\lceil n / 2\rceil$-polygon-circle representation. An example of this representation for $W_{5}$ in particular is given in Figure 1. 

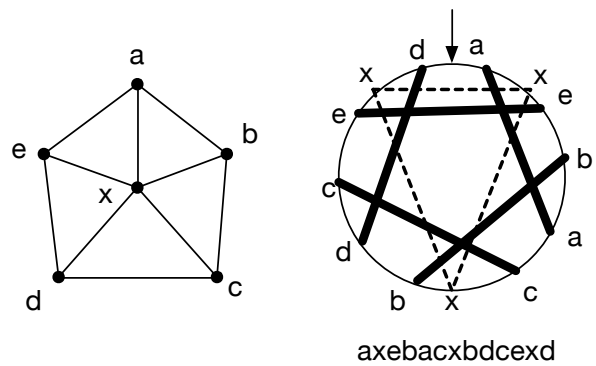

Fig. 1. The wheel $W_{5}$ with a polygon-circle representation. All vertices except for $x$ are represented with lines, and the vertex $x$ is represented by the dashed triangle. The corner-string below the representation is produced by starting at the top of the circle (indicated by the arrow) and reading clockwise around the circle.

Theorem 3.2 There are non-word-representable polygon-circle graphs.

Proof. This is an immediate corollary of Lemmas 2.4 and 3.1.

Lemma 3.3 The Petersen graph is not a polygon-circle graph.

Proof. We proceed by contradiction. Let $\mathcal{S}$ be a corner-string of a representation of the Petersen graph $P=\left(V_{P}, E_{P}\right)$, as in Figure 2: we denote the segment corresponding to vertex $v$ as $s(v)$. By Lemma 2 there is at least one segment on each of the inner and outer cycles of five vertices that contains all segments corresponding to non-neighbours. Without loss of generality, let vertex $a$ be a vertex on the outer cycle represented by a segment that is outermost and contains the segments corresponding to $d$ and $c$.

Let $N[a]$ be the closed neighbourhood of $a$. We now argue that no segment corresponding to a vertex in $V \backslash N[a]$ can contain any other: let $x, y$ be nonadjacent vertices in $V \backslash N[a]$. Both $s(x) \subset s(a)$ and $s(y) \subset s(a)$. If $s(x) \subset$ $s(y)$, then by Lemma 2.2 every path from $x$ to a must pass through the neighbourhood of $y$, a contradiction to the fact that every member of $V \backslash N[a]$ has a path to $a$ avoiding the neighbourhood of each other member of $V \backslash N[a]$.

Then no segment representing a vertex in $V \backslash N[a]$ contains any other. However, the graph $P[V \backslash N[a]]$ is a chordless cycle on six vertices, which by Lemma 2 requires containment, a contradiction.

Theorem 3.4 The are word-representable non-polygon-circle graphs.

Proof. This is an immediate corollary of Lemmas 3.3 and 2.5.

Lemma 3.5 Crown graphs $H_{n, n}$ for $n \geq 4$ are not polygon-circle graphs. 


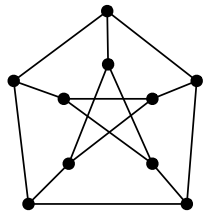

Petersen graph

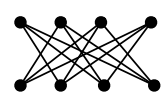

Crown $\mathrm{H}_{4,4}$

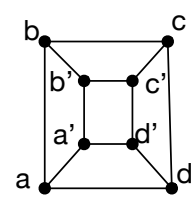

Labeled cube

Fig. 2. Three graphs that we show are not polygon-circle graphs, some labeled for convenience. Note that the crown and cube on the right are isomorphic.

Proof. Because $H_{n+1, n+1}$ contains $H_{n, n}$ as an induced subgraph, it suffices to show that $H_{4,4}$ (isomorphic to the cube) is not a polygon-circle graph. It is already known that the cube is not a member of a superclass of the polygon-circle graphs [6]. A direct argument in the polygon-circle setting is also possible, and hinges on the repeated use of Lemma 2 along with the antisymmetry of segment containment to show that representing the many 4-cycles in the cube leads to contradictory constraints.

Theorem 3.6 For every $k \geq 3$, there are $k$-word-representable graphs that are neither $(k-1)$-word-representable nor polygon-circle.

Proof. The desired result follows from Lemmas 2.6 and 3.5, since $H_{2 k, 2 k}$ is $k$-word-representable but not $(k-1)$-word-representable for $k \geq 3$.

\section{References}

[1] M. Glen, S. Kitaev, A. Pyatkin: On the representation number of a crown graph, Discrete Applied Mathematics 244 (2018) 89-93.

[2] S. Kitaev, A Comprehensive Introduction to the Theory of Word-Representable Graphs, Lecture Notes in Computer Science 10396 (2017) 36-67.

[3] S. Kitaev, V. Lozin, Words and Graphs, Springer, 2015.

[4] M. Koebe, On a new class of intersection graphs, Ann. Discrete Math. (1992) 141-143.

[5] M. Pergel, Recognition of polygon-circle graphs and graphs of interval filaments is NP-complete, Graph-Theoretic Concepts in Computer Science: 33rd International Workshop, WG 2007, Dornburg, Germany, June 21-23, 2007, Revised Papers, Lecture Notes in Computer Science, 4769 (2007) 238-247.

[6] J. Spinrad, Efficient graph representations, Fields Institute Monographs, 2003. 